\title{
Dirac-fermion-mediated ferromagnetism in a topological insulator
}

\author{
Joseph G. Checkelsky ${ }^{1 \star}$, Jianting Ye $^{2}$, Yoshinori Onose ${ }^{2,3}$, Yoshihiro Iwasa ${ }^{1,2}$ and Yoshinori Tokura ${ }^{1,2,3}$
}

\begin{abstract}
Topological insulators are a newly discovered class of materials in which helical conducting modes exist on the surface of a bulk insulator ${ }^{1-6}$. Recently, theoretical works have shown that breaking gauge symmetry ${ }^{7}$ or time-reversal symmetry ${ }^{8}$ in these materials produces exotic states that, if realized, represent substantial steps towards realizing new magnetoelectric effects $^{9,10}$ and tools useful for quantum computing ${ }^{11}$. Here we demonstrate the latter symmetry breaking in the form of ferromagnetism arising from the interaction between magnetic impurities and the Dirac fermions ${ }^{12,13}$. Using devices based on cleaved single crystals of $\mathrm{Mn}$-doped $\mathrm{Bi}_{2} \mathrm{Te}_{3-y} \mathrm{Se}_{y}$, the application of both solid-dielectric and ionic-liquid gating allows us to measure the transport response of the surface states within the bulk bandgap in the presence of magnetic ions. By tracking the anomalous Hall effect we find that the surface modes support robust ferromagnetism as well as magnetoconductance that is consistent with enhanced one-dimensional edge-state transport on the magnetic domain wall. Observation of this evidence for quantum transport phenomena demonstrates the accessibility of these exotics states in devices and may serve to focus the wide range of proposed methods for experimentally realizing the quantum anomalous Hall effect $^{8,10}$ and states required for quantum computing ${ }^{14,15}$.
\end{abstract}

The discovery of topological insulators has attracted wide interest owing to the exotic spin and charge properties of their edge modes. Among three-dimensional topological insulators, particular interest has been focused on a family of spin-orbit-coupled semiconductors based on $\mathrm{Bi}: \mathrm{Bi}_{1-x} \mathrm{Sb}_{x}, \mathrm{Bi}_{2} \mathrm{Te}_{3}$ and $\mathrm{Bi}_{2} \mathrm{Se}_{3}$ (refs 6,16,17). An active area of research since their discovery has been the breaking of time-reversal symmetry (TRS) in these systems ${ }^{12,13}$. The application of a magnetic field $B$ is predicted to give rise to a half-integer quantum Hall effect on each surface akin to graphene but with spin and valley degeneracy removed ${ }^{18}$. Alternatively, the introduction of ferromagnetism is predicted to produce a quantum anomalous Hall effect $^{8}$ (QAHE) and a topological magnetoelectric effect ${ }^{9}$, both of which are hallmarks of a topologically non-trivial state.

Original theoretical proposals involving broken TRS through ferromagnetism focused on depositing magnetic insulators on the surface of topological insulators to proximitize the surface states $^{14,15}$. More recently, it has been shown that magnetic ions substituted for $\mathrm{Bi}$ in the parent compound can realize TRS breaking even in the absence of bulk ferromagnetic ordering ${ }^{19}$. In analogy to bulk dilute magnetic semiconductors, such as $(\mathrm{Ga}, \mathrm{In})_{1-x} \mathrm{Mn}_{x} \mathrm{As}$, where hole-like carriers mediate a ferromagnetic interaction among localized spins ${ }^{20}$, one further scenario is ferromagnetic ordering mediated by the surface Dirac electrons ${ }^{12,13}$. As shown in Fig. 1a, we expect the exchange coupling $J$ between the $z$ components of the Dirac electron spin $\boldsymbol{\sigma}$ and the localized spin $\boldsymbol{S}$ to compete energetically with the Rashba-like spin texture, locking $\boldsymbol{\sigma}$ perpendicular to the momentum $\mathbf{k}$, namely the Hamiltonian $H$ has the form

$$
H=\hbar v_{\mathrm{F}}(\mathbf{k} \times \hat{\mathbf{z}}) \cdot \boldsymbol{\sigma}-J n_{\mathrm{s}} \bar{S}_{\mathrm{z}} \sigma_{\mathrm{z}}
$$

where $2 \pi \hbar$ is the Planck constant, $v_{\mathrm{F}}$ is the Fermi velocity, $\hat{\mathbf{z}}$ is the surface unit normal, $\sigma_{\mathrm{z}}$ is the $z$ component of the electron spin and $n_{\mathrm{S}}$ is the areal density of localized spins with average $z$ component $\bar{S}_{z}$ (ref. 10). Accordingly, starting from $\mathbf{k}=0$ (the Dirac point), the ground state will be TRS broken with finite $\sigma_{\mathrm{z}}$ generating an energy gap $\Delta_{\mathrm{E}}$ (representing a finite mass $m=-J n_{\mathrm{s}} \bar{S}_{\mathrm{z}}$ in the Dirac spectrum and spontaneous lowering of the electronic energy), as shown schematically in Fig. 1b. Here, we report such a ferromagnetic response through the interaction between local spins and surface Dirac electrons when the chemical potential $\mu$ lies in the bulk electronic bandgap. The observed anomalous Hall effect (AHE) and concurrent magnetoconductance stand contrary to the behaviour of conventional dilute magnetic semiconductors and are consistent with the surface-state-mediated ferromagnetism approaching the QAHE ground state.

The starting point of our study is growth of single crystals of $\mathrm{Mn}_{x} \mathrm{Bi}_{2-x} \mathrm{Te}_{3-\gamma} \mathrm{Se}_{y}$ using the Bridgman method. As shown for $x=0.04$ and $y=0.12$ (carrier density $n_{3 \mathrm{D}}=6 \times 10^{19} \mathrm{e}^{+} \mathrm{cm}^{-3}$ ) in Fig. 1c, these crystals are bulk p-type ferromagnets with the easy axis along $c$ and a transition temperature $T_{\mathrm{C}} \approx 13 \mathrm{~K}$. Also shown are the associated rise in the $B=0$ extrapolated Hall conductivity $\Delta \sigma_{x y}(T)$ (the remnant value in the case of hysteretic $\sigma_{x y}(B)$ ) that tracks the magnetization $M(T)$ and an anomaly in $\sigma_{x x}(T)\left(\sigma_{3 \mathrm{D}}\right.$ in Fig. 1c) near $T_{\mathrm{C}}$. Generally, $\sigma_{x y}$ can be decomposed as $\sigma_{x y}=\sigma_{x y}^{\mathrm{N}}+\sigma_{x y}^{\mathrm{A}}$, where $\sigma_{x y}^{\mathrm{N}}$ is due to the normal Lorentz force Hall effect and $\sigma_{x y}^{\mathrm{A}}$ is due to the AHE (ref. 21). The AHE term follows the direct proportionality $\sigma_{x y}^{\mathrm{A}}=S_{\mathrm{H}}(T) M$, demonstrated in the inset of Fig. 1d with an appropriate choice of the scaling constant $S_{\mathrm{H}}$. We will use $\Delta \sigma_{x y}$ to track $\sigma_{x y}^{\mathrm{A}}$ and $M$ also in the microcrystal-based devices where magnetization cannot be directly measured.

Our next step is to probe the surface transport properties of these crystals. Reducing the crystal thickness $t$ to between 5 and $10 \mathrm{~nm}$ greatly suppresses the bulk conductance channel and allows for manipulation of $\mu$ by electrostatic gates. As shown in Fig. 1d, crystals are cleaved onto heavily doped Si substrates with a $300 \mathrm{~nm}$ $\mathrm{SiO}_{2}$ over-layer acting as a back gate with voltage $V_{\mathrm{B}}$. Electrical contacts are made by electron beam lithography (shown in the atomic force microscope image in Fig. 1e) and finally an ionic liquid contacted in a side-gate configuration is deposited to act as a top

\footnotetext{
${ }^{1}$ Cross-Correlated Materials Research Group (CMRG) and Correlated Electron Research Group (CERG), RIKEN-ASI, Wako 351-0198, Japan, ${ }^{2}$ Department of Applied Physics and Quantum-Phase Electronics Center (QPEC), University of Tokyo, Hongo, Tokyo 113-8656, Japan, ${ }^{3}$ Multiferroics Project, ERATO, Japan Science and Technology Agency (JST), c/o University of Tokyo, Hongo, Tokyo 113-8656, Japan. *e-mail: checkelsky@riken.jp.
} 


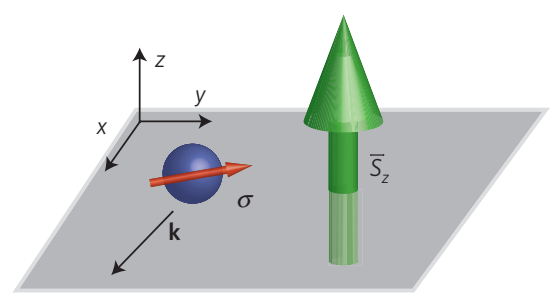

d

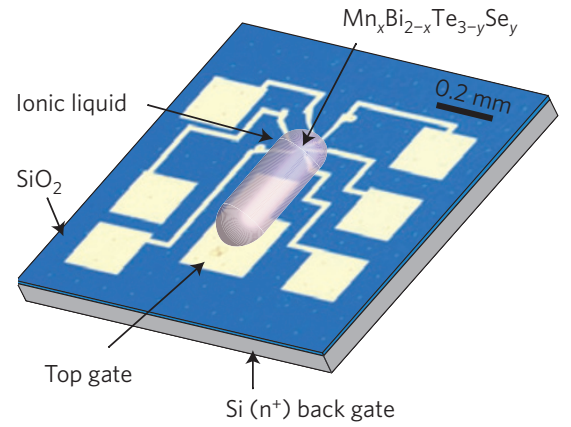

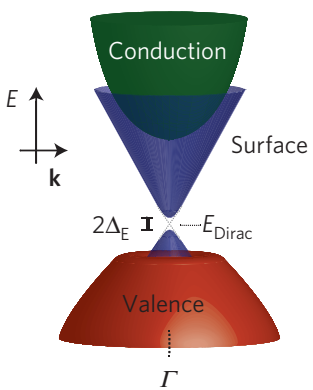

e

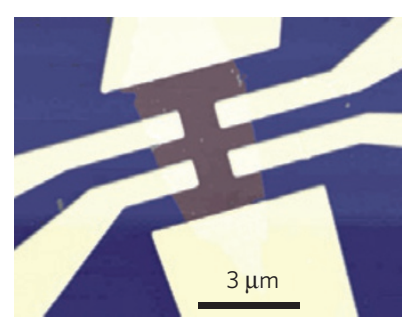

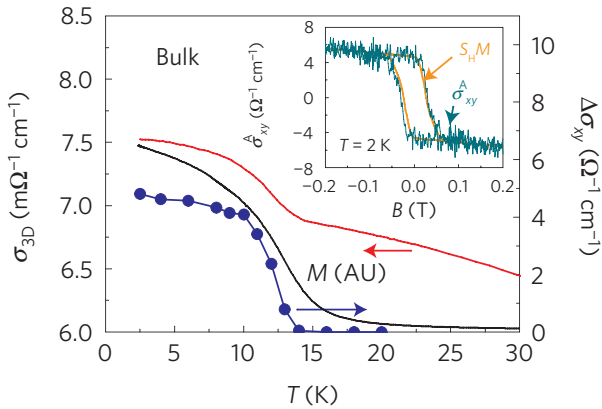

$\mathbf{f}$

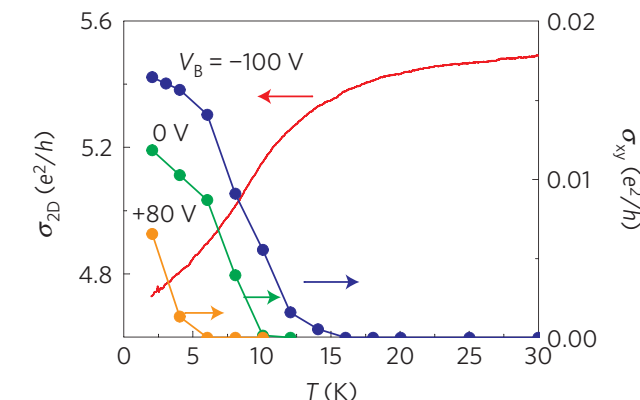

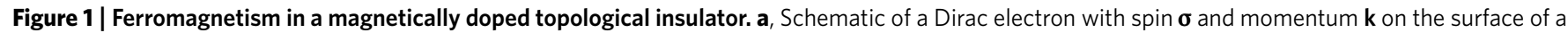
topological insulator with an average $z$ component of local spins $\bar{S}_{z}$. In the TRS broken state, the competition between the exchange interaction and the Rashba-like surface texture yields a finite component of $\boldsymbol{\sigma}$ in the $z$ direction. $\mathbf{b}$, Breaking of TRS opens a gap in the surface state spectrum starting at the Dirac point located at the time reversal invariant momentum $\Gamma$. c, Bulk crystals of $\mathrm{Mn}_{x} \mathrm{Bi}_{2-x} \mathrm{Te}_{3-y} \mathrm{Se}_{y}$ show ferromagnetic ordering below $T_{C}=13 \mathrm{~K}$, as seen in $M(T)$. Both $\sigma_{x x}$ and $\Delta \sigma_{x y}$ (the spontaneous component of $\sigma_{x y}$ ) show anomalies at $T_{C}$. The inset shows scaling of $M$ and the anomalous part of the Hall conductivity $\sigma_{x y}^{A}$. d. Device structure for measurement of microcrystals. A back gate is formed by the SiO $2 / \mathrm{Si}_{\mathrm{s}} \mathrm{substrate}$ and a top gate with the deposition of ionic liquid. e, Atomic force micrograph (false colour) of Ti/Au contacts made by electron beam lithography for device B. The crystal thickness is $7 \mathrm{~nm}$. f, Measurements of $\Delta \sigma_{x y}$ for device $A$ indicate a ferromagnetic transition. Application of the back-gate voltage $V_{B}$ is shown to tune the magnetic response with varying of the transition temperature $T_{C}$.

gate with voltage $V_{\mathrm{T}}$ (ref. 22). The act of cleavage and fabrication induces defects that tend to $\mathrm{e}^{-}$-dope devices ${ }^{23}$. With no applied gate voltage, the two-dimensional carrier density derived from $\sigma_{x y}^{\mathrm{N}}$ for device A in Fig. If is $n_{2 \mathrm{D}}=2.4 \times 10^{13} \mathrm{e}^{-} \mathrm{cm}^{-2}$. The critical value of $n_{2 \mathrm{D}}$ to reach the bottom of the conduction band is estimated to be $2 \times \mathcal{A}_{k} /(2 \pi)^{2}=2.8 \times 10^{13} \mathrm{e}^{-} \mathrm{cm}^{-2}$ using the Fermi surface area $\mathcal{A}_{k}$ observed in $\mathrm{Bi}_{2} \mathrm{Te}_{3}$ at the onset of bulk conduction ${ }^{17}$, so that $\mu$ is in the vicinity of the conduction band edge in Fig. 1b. Despite the change from the bulk crystals, in particular from p-type to n-type conduction, we observe an abrupt increase in $\Delta \sigma_{x y}(T)$, indicating a ferromagnetic transition qualitatively similar to the bulk crystals. In this class of Mn-doped compounds, n-type bulk carriers have not been observed to mediate ferromagnetism ${ }^{19,24}$, which makes the robust ferromagnetism here unexpected. Fig. If also shows that $T_{\mathrm{C}}$ can be tuned by $V_{\mathrm{B}}$, which we use below to further investigate this unconventional $n_{2 \mathrm{D}}$ dependence of the ferromagnetism.

We report the results of five devices A-E with different $V_{\mathrm{B}}$ and $V_{\mathrm{T}}$ covering the range $3 \times 10^{12} \mathrm{e}^{+} \mathrm{cm}^{-2}<n_{2 \mathrm{D}}<6 \times 10^{13} \mathrm{e}^{-} \mathrm{cm}^{-2}$, where $n_{2 \mathrm{D}}\left(V_{\mathrm{B}}\right) \equiv n_{2 \mathrm{D}}(0 \mathrm{~V})+\eta V_{\mathrm{B}}$ with the calibrated gate efficiency $\eta=8.9 \times 10^{10} \mathrm{e}^{-} \mathrm{cm}^{-2} \mathrm{~V}^{-1}$ (Supplementary Section SB and Fig. S3). $V_{\mathrm{T}}$ is fixed for each device throughout the experiment. Beginning with device A, in Fig. $2 \mathrm{a}(T=2 \mathrm{~K})$ we see an enhancement of the spontaneous $\sigma_{x y}$ with depletion of $\mathrm{e}^{-}$carriers. Starting at large positive $V_{\mathrm{B}}=+100 \mathrm{~V}\left(n_{2 \mathrm{D}}=3.3 \times 10^{13} \mathrm{e}^{-} \mathrm{cm}^{-2}\right)$, the hysteresis becomes progressively more pronounced as $V_{\mathrm{B}}$ is lowered. In Fig. $2 \mathrm{~b}$ we show the result for device $\mathrm{B}$ after application of $V_{\mathrm{T}}=-3 \mathrm{~V}$, resulting in a lower $n_{2 \mathrm{D}}=6 \times 10^{12} \mathrm{e}^{-} \mathrm{cm}^{-2}$. The growth of $\sigma_{x y}^{\mathrm{A}}$ continues with depletion of carriers, seeming to saturate at the lowest $V_{\mathrm{B}}$ where $\sigma_{x y}^{\mathrm{N}}$ changes sign. For the case of larger $n_{2 \mathrm{D}}$ where $\mu$ lies above the bulk conduction band minimum, we do not observe any sign of $\sigma_{x y}^{\mathrm{A}}$ up to $5.5 \times 10^{13} \mathrm{e}^{-} \mathrm{cm}^{-2}$ (device E). This again confirms that the bulk $\mathrm{n}$-type carriers cannot mediate ferromagnetism.
Next, the typical $T$ dependence of $\sigma_{x y}(B)$ is shown for device $\mathrm{C}$ in Fig. 2c. Here, on decreasing $T$ below $12 \mathrm{~K}$ there is an abrupt onset attributed to $\sigma_{x y}^{\mathrm{A}}$. We can estimate $T_{\mathrm{C}}$ by examining either the remnant $\Delta \sigma_{x y}(T)$ as in Fig. 1c or using an Arrott plot (Supplementary Fig. S4). In Fig. 2d, $T_{\mathrm{C}}$ for each device is plotted as a function of $n_{2 \mathrm{D}}$. The phase boundary separating the ferromagnetic and paramagnetic state is dome shaped, closing at a critical density $n_{\mathrm{c}}=(3.8 \pm 0.5) \times 10^{13} \mathrm{e}^{-} \mathrm{cm}^{-2}$, indicated in the inset of Fig. $2 \mathrm{~d}$. This finding and its implications, which we next explicate, are the main result of this paper.

In conventional dilute magnetic semiconductors, the mean field theory ordering temperature scales as $T_{\mathrm{MF}} \sim J^{2} N_{\mathrm{s}} \mathcal{D}\left(\epsilon_{\mathrm{F}}\right)$, where $N_{\mathrm{s}}$ is the number density of local spins and $\mathcal{D}\left(\epsilon_{\mathrm{F}}\right)$ is the density of states at the Fermi energy ${ }^{20,25}$. It is therefore understood that an increase in $n$ would lead to an increase in $T_{\mathrm{C}}$ (refs 26,27); this is contrary to the observation here. As expressed by equation (1), the surface Dirac electrons in the bulk bandgap mediate a ferromagnetic coupling among the local moments (Mn spins) that favours polarization of the carrier spin $\left(\sigma_{z}\right)$ and the averaged moments $\left(\bar{S}_{z}\right)$ in competition with the Rashba energy. In this way, $T_{\mathrm{C}}\left(n_{2 \mathrm{D}}\right)$ as shown in Fig. $2 \mathrm{~d}$ may be seen as primarily a reflection of the onset of TRS breaking rather than the conventional carrier number's role in ordering.

We can compare this result with the mean field value calculated for ferromagnetic ordering of magnetic impurities on the surface of a topological insulator given in ref. 12:

$$
k_{\mathrm{B}} T_{\mathrm{C}}=\frac{a_{0}^{2} S(S+1) J^{2} x}{6 \pi \hbar^{2} v_{\mathrm{F}}^{2}}(\Lambda-\mu)
$$

where $a_{0}$ is the lattice constant and $\Lambda$ is a cutoff energy associated with the termination of the Dirac surface band. Equation (2) indicates a maximum $T_{\mathrm{C}}$ at the Dirac point that smoothly decreases 

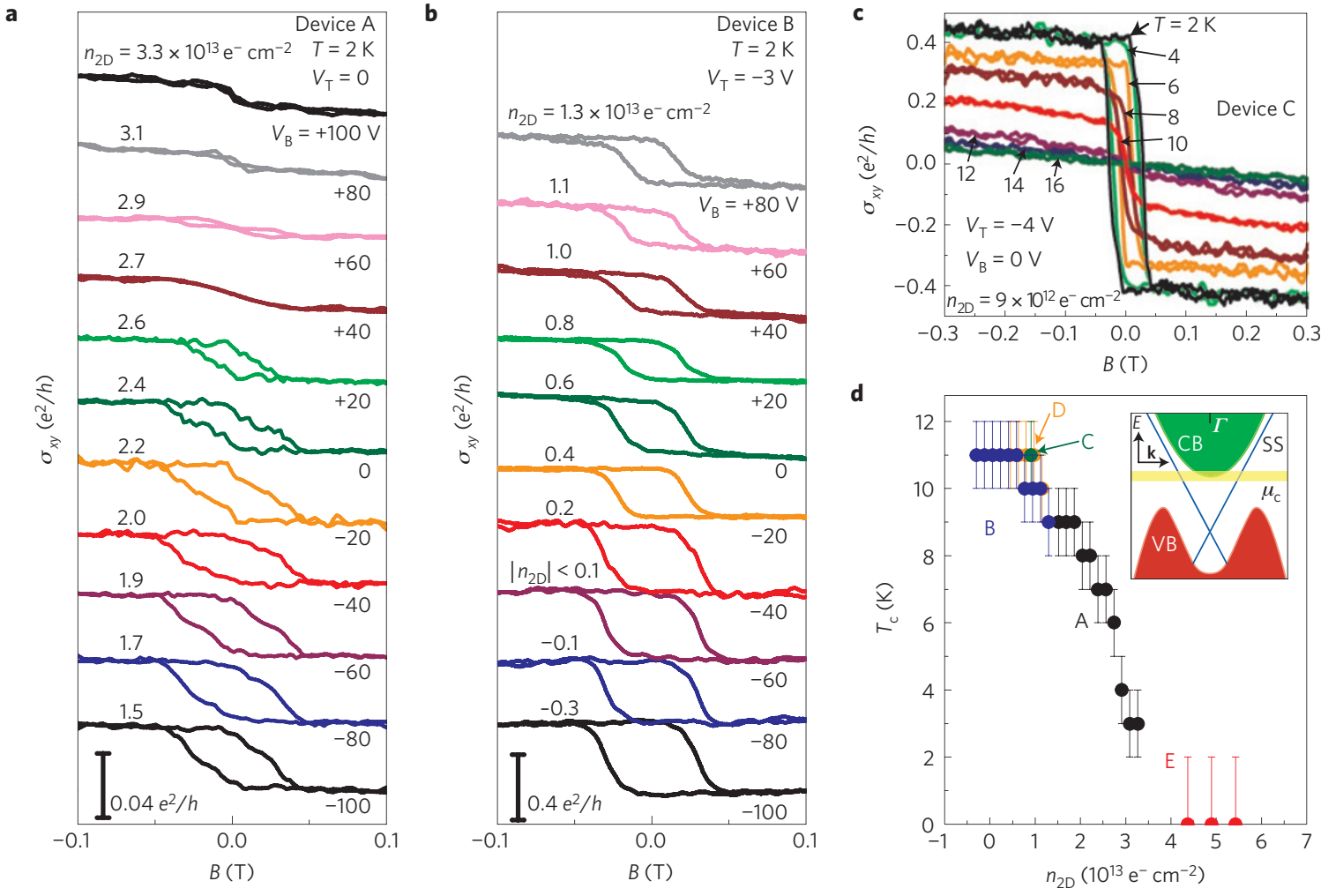

Figure 2 | AHE and $T_{C}$ tuned by carrier density. a, Hall conductivity $\sigma_{x y}$ at $T=2 \mathrm{~K}$ for device $A$ at several back-gate voltages $V_{B}$, offset vertically $0.04 e^{2} / h$. The observed anomalous Hall response grows on depletion of carriers. $\mathbf{b}$, After application of $V_{T}=-3 V$, device $B$ shows a further enhanced spontaneous $\sigma_{x y}$ whereas the ordinary Hall response $\sigma_{x y}^{N}$ changes from n-type to $p$-type at the most negative $V_{B}$. Here, a vertical offset of $0.4 \mathrm{e}^{2} / \mathrm{h}$ is used. c, Temperature dependence of $\sigma_{x y}$ in device $C$. There is a sharp onset of $\sigma_{x y}^{A}$ below $12 \mathrm{~K}$. See Fig. $1 \mathrm{f}$ for the case of device A. d, Estimates of $T_{\mathrm{C}}$ for devices A-E. Contrary to conventional dilute magnetic semiconductors, the transition is suppressed on increasing the carrier density. The error bars reflect the discreteness of the measurement in $T$. The inset depicts the critical chemical potential position $\mu_{\mathrm{c}}$ for the onset of ferromagnetic behaviour, as estimated from $\sigma_{x y}^{\mathrm{N}}$ relative to the conduction band (CB), valence band (VB) and surface states (SS).

with increasing $\mu=\hbar v_{\mathrm{F}} \sqrt{2 \pi n_{2} \mathrm{D}}$, in accord with our observation identifying $T_{\mathrm{C}}=0$ at $\mu\left(n_{\mathrm{c}}\right)=\Lambda$. Whereas equation (2) predicts a steadily increasing $T_{\mathrm{C}}$ as $\mu$ approaches the Dirac point, we observe a saturation at the lowest $n_{2 \mathrm{D}}$. This can be understood as the natural consequence of finite charge inhomogeneity in our devices. Such inhomogeneity effectively supports a residual carrier density $n^{*}$ as has been discussed in the context of the minimum conductivity at the Dirac point ${ }^{28}$. Here, from analysis of $n_{2 \mathrm{D}}\left(V_{\mathrm{B}}\right)$, we estimate $n^{*} \approx 0.5 \times 10^{13} \mathrm{e}^{-} \mathrm{cm}^{-2}$ (see Supplementary Section SB), in good agreement with the onset of saturation in Fig. 2d. Evaluating equation (2) (see Supplementary Section SC), we find $T_{\mathrm{C}} \approx 13 \mathrm{~K}$ in the vicinity of the Dirac point, within $20 \%$ of our observed value. We note that an enhanced Van Vleck susceptibility in the bulk bands has been proposed as a mechanism for generating magnetism on a comparable energy scale $^{8}$; the present results do not rule out the existence of such a phenomenon, but the agreement between equation (2) and the observed $T_{\mathrm{C}}\left(n_{2 \mathrm{D}}\right)$ strongly suggests that the surface electron scenario is relevant here.

We now turn to the $B$ dependence of the longitudinal conductivity $\sigma_{x x}$. As shown for device A in Fig. 3a, starting at $V_{\mathrm{B}}=+100 \mathrm{~V}$ (large $n_{2 \mathrm{D}}$ ) we observe the conventional butterfly pattern associated with increased scattering at domains walls ${ }^{29}$, but as we move to lower $V_{\mathrm{B}}\left(\right.$ smaller $\left.n_{2 \mathrm{D}}\right)$ we observe a sign reversal, that is, an enhanced conductivity in the vicinity of the magnetization reversal. In terms of $T$ dependence, this trend is clearly illustrated for the low/high-density regime in Fig. $3 \mathrm{~d}$ and Fig. 3e, respectively, where for $T<T_{\mathrm{C}}$ this enhancement/decrement tracks the $M$ reversal. We interpret this anomalous behaviour at low $n_{2 \mathrm{D}}$ in terms of an enhancement of a domain-wall conductance as $\mu$ approaches $\Delta_{\mathrm{E}}$. Magnetic topological insulators are anticipated to have the unique property that their domain walls trap chiral conducting modes. Viewed equivalently as either the trapped edge mode of an unfolded integer quantum Hall state ${ }^{18}$ or a bound mode analogous to solitons in doped polyacetylene ${ }^{30}$, a mode with conductance $\sigma_{\mathrm{DW}}=\sigma_{x y}^{\mathrm{A}}$ is predicted to exist along an isolated boundary of $M$ reversal. Consistent with this, the conductivity enhancement also appears in the virgin behaviour as shown in Fig. 3b. Defining $\Delta \sigma_{x x}^{\mathrm{v}}$ as the difference between the virgin and trained state at $B=0$, we see a systematic enhancement of $\Delta \sigma_{x x}^{\mathrm{v}}\left(n_{2 \mathrm{D}}\right)$ for in-gap states (Fig. 3f). In Fig. 3c we depict a hypothetical domain structure of the virgin state (or that during reversal of $M$ ) to illustrate the appearance of these chiral conducting modes along domain walls. Future experiments in devices where the domain structure can be controlled will serve to test this interpretation (see Supplementary Section SD).

Finally, we return to the detailed behaviour of $\Delta \sigma_{x y}$. Whereas equation (1) and Fig. 1b depict a disorder-free system, it has been shown that considering disorder in a magnetically doped topological insulator for $\mu$ outside the clean limit $\Delta_{\mathrm{E}}$, a finite $\sigma_{x y}^{\mathrm{A}}$ arises approaching the quantized value inside $\Delta_{\mathrm{E}}$ (ref. 10). On decreasing $n_{2 \mathrm{D}}$, a huge enhancement in $\Delta \sigma_{x y}$ is observed but we fail to obtain the quantized value $e^{2} / h$ (see Fig. 2a-c). This behaviour can be understood in terms of the role of remnant metallic conduction in parallel with the anomalous Hall surface state. Modelled as two parallel conductance channels, the observed Hall conductivity can be written as $\sigma_{x y}^{\mathrm{obs}}=\rho_{y x}^{\mathrm{S}} /\left(\left(\rho_{x x}^{\mathrm{S}}\right)^{2}+\left(\rho_{y x}^{\mathrm{S}} / \alpha\right)^{2}\right)$, where $\mathrm{S}$ denotes the contribution of the surface channel and 


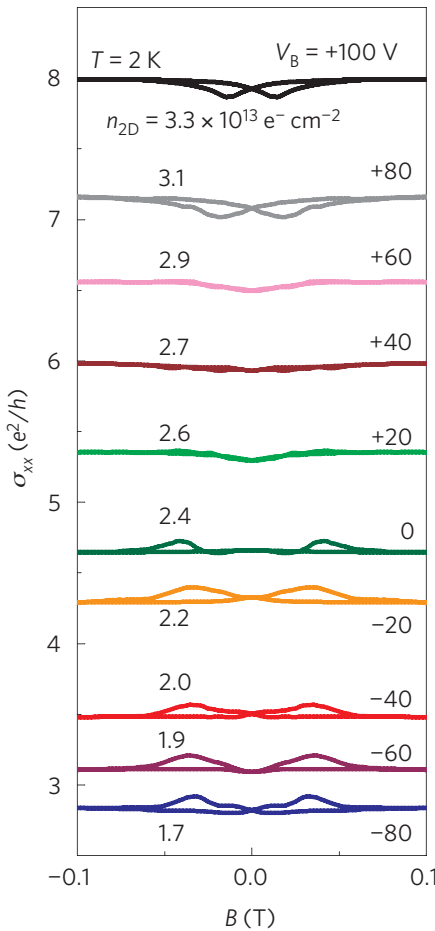

b

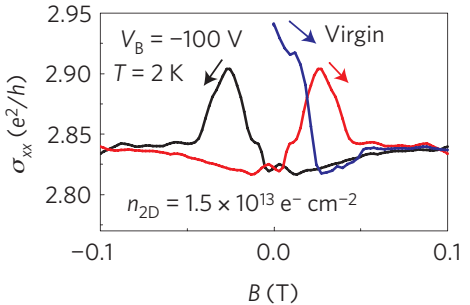

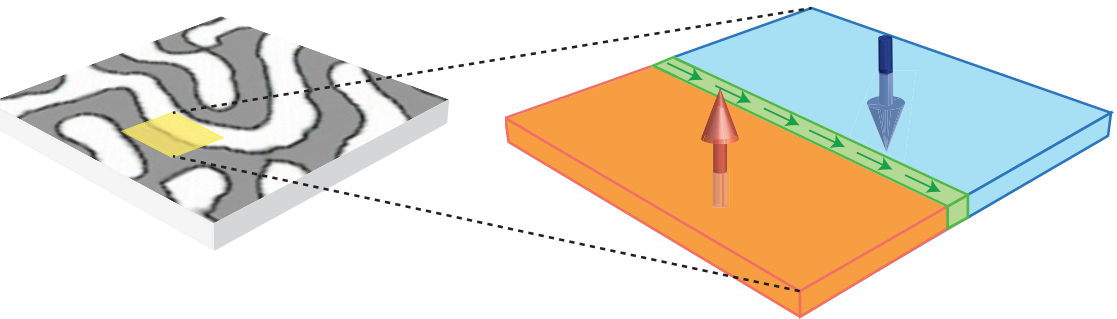

d

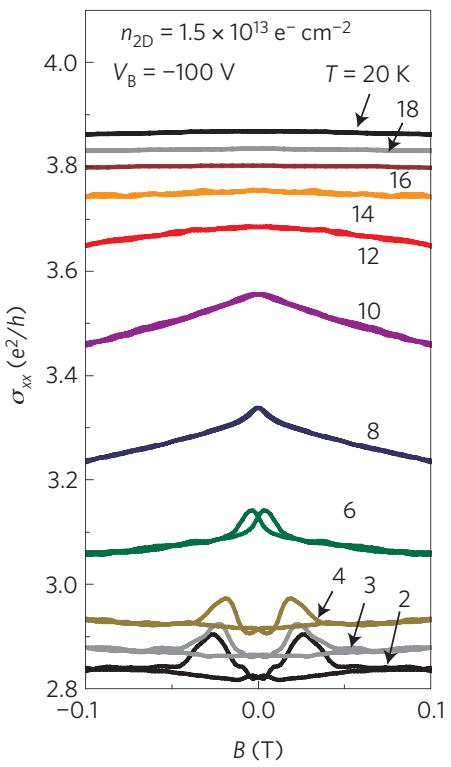

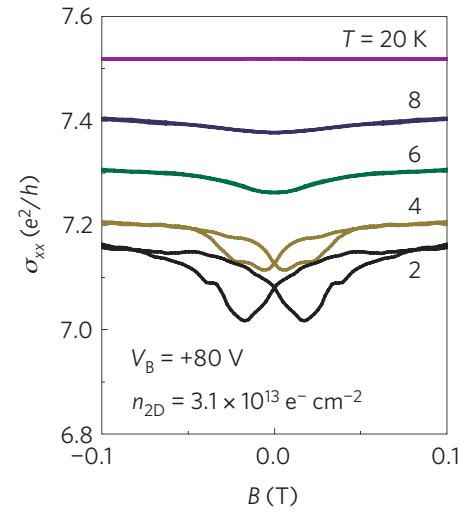

$\mathbf{f}$

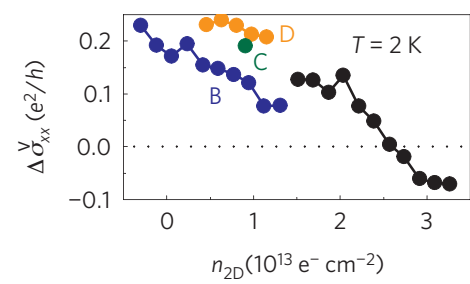

Figure 3 | Sign reversal in magnetoconductivity driven by gating for device A. a, Back-gate voltage $V_{B}$ dependence of $\sigma_{x x}$, showing the onset of enhanced conductivity at the reversal of $M$ on depletion of carriers at $T=2 \mathrm{~K}$. b. Virgin curve and trained behaviour for $V_{B}=-100 \mathrm{~V}\left(n_{2 \mathrm{D}}=1.5 \times 10^{13} \mathrm{e}^{-2} \mathrm{~cm}^{-2}\right)$. c, Schematic depiction of the domain structure in a magnetic topological insulator; domain walls across the opposite $M$ domains support a chiral mode (shown in green). d, $T$ dependence of $\sigma_{x x}(B)$ through the ferromagnetic transition gated with $V_{\mathrm{B}}=-100 \mathrm{~V}$. The enhanced conductivity can be seen to track the $M$ reversal. e, $\sigma_{x x}(B)$ at high carrier density shows hysteresis of the conventional form, that is, suppressed conductivity attributed to carrier scattering at magnetic domain walls. $\mathbf{f}$, The difference between the virgin and trained $\sigma_{x x}\left(\equiv \sigma_{x x}^{\vee}\right)$ exhibits a crossover from positive to negative values on increased carrier density.

$\alpha=\left(\rho_{x x}^{\mathrm{S}} / \rho_{x x}^{\mathrm{B}}+1\right)$ with B denoting the bulk channel. As depicted by the calculated contours of $\sigma_{x y}^{\text {obs }}$ in Supplementary Section SE, the measured Hall response is acutely sensitive to changes in $\alpha$ even for a fixed Hall contribution from the surface state. Moreover, casting the Hall response in this manner makes it apparent that the standard universal scaling of $\sigma_{x x}$ and $\sigma_{x y}^{\text {A }}$ studied in ferromagnetic systems is violated here $\mathrm{e}^{21}$. For example, holding $\rho_{y x}^{\mathrm{S}}=h / e^{2}$ and $\rho_{x x}^{\mathrm{S}}<h / e^{2}$, this simple construction shows that in fact $\sigma_{x y}^{\text {obs }}$ may overshoot and approach $e^{2} / h$ from above as the metallic channel is suppressed $\left(\rho_{x x}^{\mathrm{B}}>h / e^{2}\right)$. We expect this counterintuitive result will be ubiquitous in doped magnetic topological insulators and other non-ideal systems approaching the QAHE ground state.

With this perspective of the approach to the QAHE state, it is clear that devices prepared with further suppressed bulk states or perhaps more plausibly bulk states with lower mobility will be necessary to observe the spontaneous $e^{2} / h$ value. Despite this difficulty, the combination of an enhanced AHE for the Dirac surface modes and indications of edge transport along magnetic domains suggests the presence of the QAHE, which in principle fully develops as $T$ approaches zero ${ }^{10}$. The realization of this TRS broken state accessible by transport measurements is a significant step forward in the realization of dissipationless devices using topologically non-trivial electronic states.

\section{Methods}

Single crystals of $\mathrm{Mn}_{x} \mathrm{Bi}_{2-x} \mathrm{Te}_{3-y} \mathrm{Se}_{y}$ are grown using $99.999 \%$ pure powders of $\mathrm{Bi}$, Te and Se and a $>99.9 \%$ pure powder of MnTe. After vacuum sealing in a quartz tube, the powders are heated in a Bridgman furnace to $800^{\circ} \mathrm{C}$ and held for two days before pulling through a temperature gradient at $10^{\circ} \mathrm{C} \mathrm{cm}^{-1}$ at $2 \mathrm{~mm} \mathrm{~h}^{-1}$ for one week followed by furnace cooling. Powder X-ray diffraction is performed on the resulting crystals to confirm growth of a single phase; $x$ and $y$ are determined by energy-dispersive X-ray spectroscopy. Mn acts as a local magnetic ion replacing Bi with concentration $x$, whereas low levels of Se doping $y$ are used to compensate hole carriers arising from crystalline defects and the Mn substitution. Bulk crystals are characterized using a commercial superconducting quantum interference device magnetometer to determine magnetic properties. For transport of bulk crystals, Au wires are attached with Ag paint to apply current in the $a b$-plane and magnetic field applied along the $c$ axis. For microcrystal measurement, crystals are cleaved using Scotch tape onto cleaned $\mathrm{SiO}_{2} / \mathrm{Si}$ wafers. As described for a number of materials including $\mathrm{Bi}_{2} \mathrm{Se}_{3}$ (ref. 23), the colour of the cleaved crystal can be used to identify $t$ for crystals with $t \lesssim 30 \mathrm{~nm}$. Conventional electron beam lithography, electron beam evaporation and liftoff techniques are used to make electric contact (Ti/Au: $3 / 77 \mathrm{~nm}$ ) in a Hall bar geometry. To avoid exposure to moisture, the device is loaded into the cryostat immediately after wire bonding to the contact pads and sealing with the ionic liquid N, $N$-diethyl- $N$-(2-methoxyethyl)- $N$-methylammonium bis-(trifluoromethylsulphonyl)-imide (DEME-TFSI) as a top gate. Transport 
measurements are performed using standard four-probe a.c. methods at low frequency $(<13 \mathrm{~Hz})$ and typical excitation current $200 \mathrm{nA}$. The magnetic field is directed along the $c$ axis.

\section{Received 14 February 2012; accepted 11 July 2012; published online} 19 August 2012

\section{References}

1. Kane, C. L. \& Mele, E. J. Z2 topological order and the quantum spin Hall effect. Phys. Rev. Lett. 95, 146802 (2005).

2. Bernevig, B. A., Hughes, T. L. \& Zhang, S-C. Quantum spin Hall effect and topological phase transition in HgTe quantum wells. Science 314, 1757-1761 (2006).

3. Konig, M. et al. Quantum spin Hall insulator state in HgTe quantum wells. Science 318, 766-770 (2007).

4. Fu, L., Kane, C. L. \& Mele, E. J. Topological insulators in three dimensions. Phys. Rev. Lett. 98, 106803 (2007).

5. Moore, J. E. \& Balents, L. Topological invariants of time-reversal-invariant band structures. Phys. Rev. B 75, 121306(R) (2007).

6. Hsieh, D. et al. A topological Dirac insulator in a quantum spin Hall phase. Nature 452, 970-974 (2008).

7. Linder, J., Tanaka, Y., Yokoyama, T., Sudbo, A. \& Nagaosa, N. Unconventional superconductivity on a topological insulator. Phys. Rev. Lett. 104, 067001 (2010)

8. Yu, R. et al. Quantized anomalous Hall effect in magnetic topological insulators. Science 329, 61-64 (2010).

9. Qi, X-L., Hughes, T. L. \& Zhang, S-C. Topological field theory of time-reversal invariant insulators. Phys. Rev. B 78, 195424 (2008).

10. Nomura, K. \& Nagaosa, N. Surface-quantized anomalous Hall current and the magnetoelectric effect in magnetically disordered topological insulators. Phys. Rev. Lett. 106, 166802 (2011).

11. Fu, L. \& Kane, C. L. Superconducting proximity effect and Majorana fermions at the surface of a topological insulator. Phys. Rev. Lett. 100, 096407 (2008).

12. Liu, Q., Liu, C-X., Xu, C., Qi, X-L. \& Zhang, S-C. Magnetic impurities on the surface of a topological insulator. Phys. Rev. Lett. 102, 156603 (2009).

13. Abanin, D. A. \& Pesin, D. A. Ordering of magnetic impurities and tunable electronic properties of topological insulators. Phys. Rev. Lett. 106, 136802 (2011).

14. Fu, L. \& Kane, C. L. Probing neutral majorana fermion edge modes with charge transport. Phys. Rev. Lett. 102, 216403 (2009).

15. Akhmerov, A., Nilsson, J. \& Beenakker, C. Electrically detected interferometry of Majorana fermions in a topological insulator. Phys. Rev. Lett. 102, 216404 (2009)

16. Xia, Y. et al. Observation of a large-gap topological-insulator class with a single Dirac cone on the surface. Nature Phys. 5, 398-402 (2009).

17. Chen, Y. L. et al. Experimental realization of a three-dimensional topological insulator, $\mathrm{Bi}_{2} \mathrm{Te}_{3}$. Science 325, 178-181 (2009).

18. Hasan, M. Z. \& Kane, C. L. Colloquium: Topological insulators. Rev. Mod. Phys. 82, 3045-3067 (2010)
19. Chen, Y. L. et al. Massive Dirac fermion on the surface of a magnetically doped topological insulator. Science 329, 659-662 (2010).

20. Dietl, T., Haury, A. \& Merle d' Aubigne, Y. Free carrier-induced ferromagnetism in structures of diluted magnetic semiconductors. Phys. Rev. B 55, 3347-3350(R) (1997).

21. Nagaosa, N., Sinova, J., Onoda, S., MacDonald, A. H. \& Ong, N. P. Anomalous Hall effect. Rev. Mod. Phys. 82, 1539-1592 (2010).

22. Ye, J. T. et al. Liquid-gated interface superconductivity on an atomically flat film. Nature Mater. 9, 125-128 (2010).

23. Checkelsky, J. G., Hor, Y. S., Cava, R. J. \& Ong, N. P. Bulk band gap and surface state conduction observed in voltage-tuned crystals of the topological insulator $\mathrm{Bi}_{2} \mathrm{Se}_{3}$. Phys. Rev. Lett. 106, 196801 (2011).

24. Choi, J. et al. Single crystal growth and magnetic properties of Mn-doped $\mathrm{Bi}_{2} \mathrm{Se}_{3}$ and $\mathrm{Sb}_{2} \mathrm{Se}_{3}$. J. Magn. 9, 125-127 (2004).

25. Dietl, T., Ohno, H., Matsukura, F., Cibert, J. \& Ferrand, D. Zener model description of ferromagnetism in zinc-blende magnetic semiconductors. Science 287, 1019-1022 (2000)

26. Ohno, H. et al. Electric-field control of ferromagnetism. Nature 408, 944-946 (2000).

27. Chiba, D., Matsukura, F. \& Ohno, H. Electric-field control of ferromagnetism in (Ga,Mn)As. Appl. Phys. Lett. 89, 162505 (2006).

28. Kim, D. et al. Surface conduction of topological Dirac electrons in bulk insulating $\mathrm{Bi}_{2} \mathrm{Se}_{3}$. Nature Phys. 8, 460-464 (2012).

29. Levy, P. M. \& Zhang, S. Resistivity due to domain wall scattering. Phys. Rev. Lett. 79, 5110-5113 (1997).

30. Su, W. P., Schrieffer, J. R. \& Heeger, A. J. Solitons in polyacetylene. Phys. Rev. Lett. 42, 1698-1701 (1979).

\section{Acknowledgements}

We are grateful to N. Nagaosa, M. Kawasaki, F. Zhang, K. Nomura, B-J. Yang and S. Bahramy for fruitful discussions. This research is supported by the Japan Society for the Promotion of Science through the Funding Program for World-Leading Innovative R\&D on Science and Technology (FIRST Program), initiated by the Council for Science and Technology Policy.

\section{Author contributions}

J.G.C. and Y.O. performed the single-crystal growth and experiments. J.G.C. and J.Y. made devices and performed device experiments aided by Y.O. J.G.C. analysed the data and wrote the manuscript with contributions from all authors. Y.I. and Y.T. contributed to discussion of the results and guided the project. Y.T. conceived and coordinated the project.

\section{Additional information}

Supplementary information is available in the online version of the paper. Reprints and permissions information is available online at www.nature.com/reprints. Correspondence and requests for materials should be addressed to J.G.C.

\section{Competing financial interests}

The authors declare no competing financial interests. 